\title{
Human orthopedic research articles convey information better than veterinary orthopedic research articles: Evidence-based manuscript writing guidelines
}

Richard Evans ( $\square$ rbevans@syr.edu )

Thomas Jefferson University https://orcid.org/0000-0002-8727-3903

Antonio Pozzi

University of Zurich

\section{Research Article}

Keywords: orthopedics, surgery, writing, veterinary

Posted Date: February 1st, 2022

DOI: https://doi.org/10.21203/rs.3.rs-1309416/v1

License: (c) (i) This work is licensed under a Creative Commons Attribution 4.0 International License. Read Full License 


\section{Abstract}

\section{Objective.}

The objective of this study was to compare the brevity and clarity of human orthopedic articles to veterinary orthopedic articles. The goal is to provide authors and reviewers with objective, evidence-based guidelines to critically evaluate the brevity and clarity of veterinary manuscripts during the writing phase of research and the review process.

\section{Study design.}

We used word counts and the percent of active voice sentences to assess the brevity and clarity of the introduction sections and discussion sections in 15 randomly chosen veterinary orthopedic clinical trial articles and 15 randomly chosen human orthopedic clinical trial articles.

\section{Results.}

Veterinary introduction sections were on average 193 words longer than human introduction sections $(p=0.001)$. Veterinary discussion sections were on average 370 words longer than human discussion sections.

Veterinary introduction sections had on average 14.4 percent fewer active voice sentences than human introduction sections $(p=0.003)$. Veterinary discussion sections had on average 8.3 percent fewer active voice sentences than human discussion sections.

\section{Conclusion.}

Human orthopedic clinical trial articles are more concise and direct than veterinary clinical trial articles.

\section{Introduction}

Direct, concise scientific articles can increase reader confidence and encourage interdisciplinary collaboration. $(1,2)$ Readers cite direct, concise articles more often than longer, less directly written articles. (2) Reviewers may read more carefully, be less distracted, and write a more focused review for shorter, succinct text. 
Two attributes help make scientific writing direct and concise. The first attribute is brevity. Brevity is important because longer introduction and discussion sections have more ideas for readers to process, and that comes at the expense of clarity. $(3,4)$ We measured brevity objectively with word counts.

The second attribute is the active voice, which improves clarity by making points directly. $(4,5,6)$ For example, "The dogs walked over the force plate," is more quickly understood than the passive voice, "The force plate was walked over by the dogs." Neuroimaging has shown that human brains require more activation to understand passive voice sentences than active voice sentences. (7)

If veterinary authors compared word counts and percent of active voice sentences in orthopedic manuscripts to those in published veterinary orthopedic articles and human orthopedic articles, then they could objectively assess if their manuscripts are more-or-less direct and concise relative to other articles.

In other words, is a 500 -word introduction section, with $40 \%$ of its sentences written in the active voice, direct and concise compared to published articles? This article addresses direct and concise writing with two aims.

The first aim is to establish baseline word counts for introduction and discussion sections in veterinary orthopedic articles, and compare word counts in veterinary orthopedic articles to word counts in human orthopedic articles.

The second Aim is to establish the baseline percent of active voice sentences in introduction and discussion sections for veterinary orthopedic articles and compare the percent in veterinary orthopedic articles to the percent in human orthopedic articles.

\section{Materials \& Methods}

\section{Study design and case selection.}

This was a two-group, cross-sectional study using veterinary orthopedic articles from Veterinary Comparative Orthopedics and Traumatology and Veterinary Surgery, and human orthopedic articles from the Journal of Bone and Joint Surgery.

To select veterinary articles, we generated lists from the Veterinary Comparative Orthopedics and Traumatology and Veterinary Surgery websites by searching on "orthopedic" and "randomized", and then refined the lists to prospective randomized clinical trials. We alternated between the lists, working down them, flipping a coin to randomly select articles for analysis.

To select human orthopedic articles, we generated a list from the Journal of Bone and Joint Surgery by searching on "randomized," and then refined the list to prospective randomized clinical trials. To randomly select articles for analysis, we worked down the list, flipping a coin. 
We removed citation numbers, headings, figures, and tables from the introduction and discussion sections before the text analysis.

\section{Outcome measures.}

The co-primary outcomes were the word counts of the introduction sections and the percent of passive voice sentences in the introduction sections. They were the only outcomes that were pre-specified for statistical testing, to guard against picking and choosing statistically significant results.

The secondary outcomes were the word counts of the discussion sections and the percent of passive voice sentences in the discussion sections

We measured outcomes using Microsoft Word Editor Insights.

\section{Sample size and the early stopping rule.}

We calculated the sample size using the approach recommended by the National Institutes of Health, arriving at 60 articles in total. (8) Processing articles for analysis is tedious and time consuming, so we used the clinical trial technique of early stopping rules to end the study early if there was a dramatic difference between the veterinary and human articles halfway through the study.

In brief, this approach allowed for a predetermined interim inferential data analysis at 30 articles on the condition that the cutoff for statistical significance is set below 0.05 . This is to accommodate the Type I error inflation caused by statistically testing the data twice: first at 30 articles, and if need be, again at 60 articles. The lower-than-0.05 cutoff for statistical significance is called the stopping rule.

We used the O'Brien and Fleming method to determine the stopping rule, stopping the study early if both p-values for the co-primary outcomes were less than 0.0054 . $(9,10)$ If either $p$-value was equal to or larger than 0.0054 , then the data collection would continue to 60 articles.

\section{Statistical analysis.}

We assessed the data for spurious observations using boxplots, and we used the Wilk-Shapiro test to test the outcomes for normality. In addition, the percent data was checked to satisfy the "npq" normal approximation.

Welch's t-test was used to statistically test introduction section word count and introduction percent active voice between veterinary and human groups of articles.

To declare one group statistically more direct and concise than another group, both p-values had to be below 0.0054 at the interim analysis.

We presented the data using Cohen's effect size and summaries as: average (standard error). 


\section{Results}

At the 30-article interim data analysis, Both the introduction word-count $p$-value $(p=0.001)$ and the percent active voice $(p=0.003)$ were less than 0.0054 . That satisfied the stopping rule, so we terminated data collection.

We analyzed 15 veterinary articles (from 2005 to 2021) and 15 human orthopedic articles (from 2004 to 2021). For the veterinary articles, one studied anesthesia, one studied gloving, and three assessed pain control. The rest studied intervention effects on the spine or joints. One studied felines, another used felines and canines, and the rest used canines or canine cadavers.

For the human articles, one studied anesthesia, one assessed opioid use, one telemedicine, and one webbased rehabilitation. The rest studied intervention effects on the spine or joints. All studied live patients.

There were no spurious observations, and all the outcomes and groups had approximately normal distributions.

The average introduction word count for veterinary articles was 522 (37.5), and for human articles it was 329 (23.7). Cohen's effect size was 1.6. As mentioned above, the population mean introduction word counts were statistically different ( $p=0.001$, using 0.0054 as the cutoff, with 23.7 degrees of freedom). The average discussion word count for veterinary articles was 1262 (83.6), and for human articles it was 892 (60.0). Cohen's effect size was 1.3.

The average introduction percent of active voice sentences for veterinary articles was $63.5 \%$ (3.41\%), and for human articles it was $77.9 \%$ (2.69\%). Cohen's effect size was 1.3. As mentioned above, the population mean introduction percent of active voice sentences were statistically different ( $p=0.003$, using 0.0054 as the cutoff, with 26.5 degrees of freedom). The average discussion percent of active voice sentences for veterinary articles was $62.3 \%(1.69 \%)$, and for human articles it was $70.6 \%(2.75 \%)$. Cohen's effect size was 0.9 .

\section{Discussion}

Human orthopedic introductions were more direct and concise than veterinary orthopedic introductions. Veterinary abstracts and discussions combined were on average 563 words longer, that is, over a page longer than human orthopedic introductions and conclusions combined.

We informally assessed causes for the difference in word count. It appeared that the veterinary articles provided more background and ancillary information than provided in human articles. Both introductions and discussions had elements similar to review papers, rather than focused explanations of the gaps in literature and a narrow discussion of the results.

Veterinary articles sometimes have more redundancies. For example, the human introductions stated aims, but veterinary articles often stated both aims and hypotheses. From the point of view of the reader, 
providing general hypotheses after stating aims can be redundant.

The veterinary introductions had $22.6 \%$ fewer active voice sentences than the human introductions, and most veterinary introductions had fewer active sentences than most human articles. For perspective, one human article introduction in this study had $100 \%$ active sentences.

However, sometimes authors prefer the passive voice to emphasize the object of a sentence rather than the subject. For example, the passive sentence, "The force plate was walked over by the dogs," emphasizes the force plate.

Still, authors could emphasize the force plate actively with, "The force plate was used to assess ground reaction forces. Dogs walked over it five times."

Sometimes writing in the active voice also requires the first-person voice, "I" or "we," because the scientist performed the action: "We walked the dogs over the force plate." In the past, scientific writing experts discouraged the first person because it placed emphasis on the authors. But does that matter? Consider the clarity of the first sentence of Watson and Crick (1953), "We wish to suggest a structure for the salt of deoxyribose nucleic acid (D.N.A.). (11) That first-person, active voice sentence is clear compared to the passive alternative, "A structure for the salt of deoxyribose nucleic acid (D.N.A.) is suggested in this paper."

Contemporary readers prefer the first person because it is easier to read. Important scientific journals, such as Nature, and the British Medical Journal prefer using "I" and "we" to awkward, passive wording that reduces clarity. $(3,4,12)$ An active voice is easier to understand for readers whose first language is not English. (12)

We acknowledge that the active voice is only one aspect of clarity. There are subjective attributes, such as noun clustering, organization, and repetition, but they can be hard to assess and quantify. (5)

This study focused on the introduction and discussion sections because they offer more opportunity for direct and concise writing than materials and methods sections, and results sections. Informally, the materials and methods sections and results sections of the human and veterinary articles appeared to have the same structures and terse reporting style. In other words, the human articles did not shorten their introductions by leaking introduction information into the materials and methods sections.

Different study designs and research topics might by necessity have considerably different introduction and discussion lengths. For example, medical articles involving public policy can have very long discussion sections. That is why we focused on a specific kind of study, orthopedic randomized clinical trials, to control variation.

The community of veterinary orthopedic clinical trial authors is relatively small compared to human authors. Some veterinary authors co-authored more than one veterinary article. There is no way to assess 
the correlated-author effect on outcomes without designing a study to do so. No author appeared as lead author more than once.

\section{Conclusions}

The objective of this study was to provide a way for authors and reviewers to critically evaluate the clarity and brevity of manuscripts. Authors of veterinary orthopedic articles should consider writing articles with less background information and with more active voice sentences.

\section{References}

1. Freeling BS, et al. Better Writing in Scientific Publications Builds Reader Confidence and Understanding. Front Psychol. 2021 Aug 27;12:714321. doi: 10.3389/fpsyg.2021.714321.

2. Hillier A, Kelly RP, Klinger T. Narrative Style Influences Citation Frequency in Climate Change Science. PLoS One. 2016 Dec 15;11(12):e0167983. doi: 10.1371/journal.pone.0167983.

3. Freeling B, Doubleday ZA, Connell SD. Opinion: How can we boost the impact of publications? Try better writing. Proc Natl Acad Sci U S A. 2019 Jan 8;116(2):341-343. doi: 10.1073/pnas.1819937116. PMID: 30622212; PMCID: PMC6329950.

4. Nature Editors 2011. How to Write Your Paper. Available at https://www.nature.com/natureportfolio/for-authors/write (accessed 01 December 2021).

5. Crichton M. Sounding board: medical obfuscation: structure and function. N Engl J Med. 1975 Dec 11;293(24):1257-9. doi: 10.1056/NEJM197512112932413. PMID: 1186808.

6. University of California staff. 2021. Scientific Writing: Active and Passive Voice. Available at https://gwc.ucr.edu/document/scientific-writing-active-and-passive-voice (accessed 22 November 2021).

7. Feng, S., Legault, J., Yang, L., Zhu, J., Shao, K., \& Yang, Y. 2015. Differences in grammatical processing strategies for active and passive sentences: An fMRI study. Journal of Neurolinguistics, 33, 104-117.

8. National Center of Complementary and Integrative Health staff 2021. Pilot Studies: Common Uses and Misuses. Available at https://www.nccih.nih.gov/grants/pilot-studies-common-uses-andmisuses (accessed 02 December 2021).

9. Hamasaki T, Asakura K, Evans SR, Sugimoto T, Sozu T. Group-Sequential Strategies in Clinical Trials with Multiple Co-Primary Outcomes. Stat Biopharm Res. 2015;7(1):36-54. doi: 10.1080/19466315.2014.1003090. PMID: 25844122; PMCID: PMC4382106.

10. Pennsylvania State University staff. 2021. Frequentist Methods: O'Brien-Fleming, Pocock, HaybittlePeto: STAT 509. Available at https://online.stat.psu.edu/stat509/lesson/9/9.5 (accessed 07 November 2021)

11. Watson JD, Crick FH. Molecular structure of nucleic acids; a structure for deoxyribose nucleic acid. Nature. 1953 Apr 25;171(4356):737-8. doi: 10.1038/171737a0. PMID: 13054692. 
12. British Medical Journal editors. 2021. Resources for Authors, House style. Available at http://resources.bmj.com/bmj/authors/bmj-house-style (accessed 07 November 2021)

\section{Declarations}

Competing interests: The authors declare no competing interests. 